\title{
Avaliação do conhecimento dos profissionais e estudantes de saúde sobre descarte adequado de medicamentos
}

\author{
Assessment of knowledge of professionals andhealth students on proper disposal \\ ofmedicines
}
Evaluación del conocimiento de profesionales yestudiantes de salud en la eliminación medicamentos

Fabiano Rodrigues Bucci ${ }^{1 *}$, Tuanny Caroline Pereira de Santana ${ }^{1}$, Manoela Almeida Santos da Figueira ${ }^{1}$, Ítala Morgânia Farias da Nóbrega ${ }^{1}$, Flávia Patrícia Morais de Medeiros¹.

\section{RESUMO}

Objetivo: Avaliar o conhecimento dos profissionais e estudantes de saúde sobre descarte adequado de medicamentos. Métodos: Trata-se de um estudo de validação, descritivo, desenvolvido em quatro etapas. A primeira etapa foi a validação de conteúdo; a segunda, foi a validação semântica; a terceira, foi a validação consensual final e a quarta etapa foi a aplicação dos questionários validados. O estudo foi realizado entre abril de 2018 a dezembro de 2019, aprovado com no de parecer 3.231.070, conforme Resolução 510/2016. Responderam os questionários $53,65 \%$ dos profissionais e $10 \%$ dos estudantes de saúde. Resultados: Destes participantes, $53,64 \%$ dos profissionais e $62,86 \%$ dos estudantes descartam medicamentos de forma inapropriada no lixo comum; $87,27 \%$ dos profissionais e $87,43 \%$ dos estudantes afirmam não conhecer as legislações sanitárias e ambientais vigentes sobre descarte de medicamentos, e somente, $7,27 \%$ entre os profissionais e $4,57 \%$ entre os estudantes dizem possuir conhecimento para orientar as pessoas como descartar medicamentos de forma adequada. Conclusão: $O$ estudo aponta que a maioria dos profissionais e estudantes de saúde participantes ainda não possuem conhecimento sobre descarte adequado de medicamentos.

Palavras-chave: Meio ambiente, Educação ambiental, Educação em saúde, Resíduos de serviços de saúde, Contaminação ambiental.

\begin{abstract}
Objective: To evaluate the knowledge of health professionals and students on the proper disposal of medicines. Methods: This is a descriptive, validation study, developed in four stages. First stage was content validation, second stage was semantic validation, third stage was final consensual validation and fourth stage was the application of validated questionnaires. The study was carried out between April 2018 and December 2019, approved with opinion number $3,231,070$, according to Resolution $510 / 2016$. $53.65 \%$ of professionals and $10 \%$ of health students from the institutions participated. Results: Of these, $53.64 \%$ of professionals and $62.86 \%$ of students dispose of medicines inappropriately in the common garbage; $87.27 \%$ of the professionals and $87.43 \%$ of the students affirm that they do not know the sanitary and environmental laws in force on the disposal of medicines, and only $7.27 \%$ among the professionals and $4.57 \%$ among the students say they have knowledge to guide the people how to dispose of drugs properly. Conclusion: The study points out that the majority of participating health professionals and students still do not have knowledge about proper drug disposal.
\end{abstract}

Keywords: Environment, Environmental education, Health education, Health services waste, Environmental contamination.

${ }^{1}$ Faculdade Pernambucana de Saúde (FPS), Recife - PE. *E-mail: fabianorbucci@gmail.com 


\section{RESUMEN}

Objetivo: Evaluar los conocimientos de los profesionales y estudiantes de la salud sobre la correcta disposición de los medicamentos. Métodos: Se trata de un estudio descriptivo y de validación, desarrollado en cuatro etapas. La primera etapa fue la validación de contenido, la segunda etapa fue la validación semántica, la tercera etapa fue la validación consensuada final y la cuarta etapa fue la aplicación de cuestionarios validados. El estudio se realizó entre abril de 2018 y diciembre de 2019, aprobado con dictamen número 3.231.070, según Resolución 510/2016. Participaron el 53,65\% de los profesionales y el $10 \%$ de los estudiantes de salud de las instituciones. Resultados: De estos, el $53,64 \%$ de los profesionales y el $62,86 \%$ de los estudiantes desechan los medicamentos de forma inapropiada en la basura común; El 87,27\% de los profesionales y el $87,43 \%$ de los estudiantes afirman no conocer las leyes sanitarias y ambientales vigentes en materia de disposición de medicamentos, y solo el $7,27 \%$ de los profesionales y el $4,57 \%$ de los estudiantes manifiesta tener conocimientos para orientar personas cómo deshacerse de las drogas correctamente. Conclusión: El estudio señala que la mayoría de los profesionales de la salud y estudiantes participantes aún no tienen conocimientos sobre la correcta eliminación de los medicamentos.

Palabras clave: Medio ambiente, Educación ambiental, Educación en salud, Servicios de salud residuos, Contaminación ambiental.

\section{INTRODUÇÃO}

No Brasil, a destinação final de medicamentos vencidos, inutilizados e sobras de tratamentos, tem gerado problemas de interfaces complexas e com enorme dificuldade de solução, pois tem sido realizada por grande parte da sociedade em destinos considerados inadequados. Atitudes essas que acarretam contaminação da água, solo, animais e danos à saúde da população (TAVERA S, et al., 2017).

A realidade de saúde moderna, onde se encontra o uso frequente de medicamentos, tem contribuído para essa prática que agride de forma significativa o meio ambiente, de modo que as legislações sobre o comércio, prescrição e uso têm sido ineficazes para minimizar os prejuízos dela decorrentes (OLIVEIRA NR, 2015).

Ramos HMP, et al. (2017) esclarecem que são incontestáveis os benefícios à saúde das pessoas com as inovações de tratamentos, gerando melhoria da qualidade de vida, combate a enfermidades e redução dos índices de morbimortalidade. Por outro lado, isso acarreta considerável aumento na quantidade de medicamentos, gerando enorme quantidade de resíduos desses produtos (GUERRIERI FM e HENKES JA, 2017).

Acrescenta-se ainda o uso irracional de medicamentos por grande parte da população; a falta de comercialização de quantidade fracionada; a distribuição de amostras grátis e o incentivo ao consumo pela mídia. Fatores esses que contribuem para que os medicamentos expirem o prazo de validade e corrobore com o acúmulo desses resíduos químicos em domicílio (RAMOS HMP, et al., 2017).

De acordo com Borges RM, et al. (2016), a presença desses resíduos nas estações de tratamentos de água representa desafio para as companhias de saneamento. Muitas substâncias não são eliminadas nas estações de tratamentos de água, podendo levar a ocorrência de contaminação, afetando animais e impactando a saúde da humanidade (ALBANAZ HF, et al., 2017).

No cenário atual predominam a desinformação da sociedade sobre a temática ambiental e a falta de implementação do recolhimento dos resíduos de medicamentos pelas farmácias, drogarias e unidades de saúde (FERREIRA CL, et al., 2015).

De acordo com Alvarenga LSV e Nicoletti MA (2010), o descarte de medicamentos pelo consumidor final em nosso país é o que apresenta maior lacuna na legislação. Isso se deve, entre outros fatores, a ineficiência de infraestrutura. Faltam aterros sanitários e incineradores adequados em grande parte do território nacional, o que compromete a adoção de medidas que possam ao menos atenuar o problema (TAVERA S, et al., 2017).

Dessa forma, tanto o uso quanto o descarte de medicamentos, devem seguir critérios mais rígidos. Os resíduos desses produtos são classificados como químicos pela Resolução no 358/2005 do CONAMA e pela 
Resolução da Diretoria Colegiada ํㅡ 222/2018 da ANVISA, devendo receber manejo, tratamento e destinação final adequada. No entanto, segundo Hoppe TR e Araújo LEB (2012), o tratamento e a destinação final dos resíduos, ainda se limita a adoção de soluções imediatas, que geralmente se restringem ao simples descarte contribuindo para a destruição ambiental.

Nesse contexto, reveste-se de importância a educação ambiental, entendida como sendo o caminho pelo qual as sociedades montam seus valores, por meio de conhecimentos, habilidades e atitudes, que ensejam a preservação ambiental, tendo a sustentabilidade como fator indispensável para a saúde. Deve ser um recurso contínuo de aprendizagem, alcançando os diversos ciclos do ensino (LEMES EO e SILVA JR, 2014).

Ademais, de acordo com Dinardi AJ e Araújo SC (2017), em diversos cursos de graduação da área de saúde não há a devida inquietude com a questão ambiental. Percebe-se que desde a elaboração dos Projetos Pedagógicos dos Cursos (PPC), vê-se que muitas vezes atemática socioambiental não é contemplada.

Devido a isso, surgem fragilidades quanto à informação gerenciamento e gestão do descarte de medicamentos, gerando dúvida na população, nos profissionais de saúde e nos estudantes (futuros profissionais), de onde e como descartar adequadamente esses produtos. Assim, é preciso que ocorra maior orientação, tanto a população em geral, mas primordialmente aos profissionais e estudantes de saúde, pois são fundamentais para esclarecer e conscientizar a população (BATISTA RS, et al., 2009).

Espera-se uma mudança de visão quanto a necessidade de gerenciamento de resíduos por parte da sociedade. Essa deve exigir do poder público a elaboração de novas legislações ambientais, que permitam novos padrões de serviço, possibilitando que os consumidores de medicamentos possam devolver os resíduos para descarte ambientalmente correto, efetivando assim a logística reversa de medicamentos no país (FEITOSA AV e AQUINO MD, 2016; TAVERA S, et al., 2017).

Portanto, o estudo teve como objetivo avaliar o conhecimento dos profissionais e estudantes de saúde sobre descarte adequado de medicamentos com questionários validados.

\section{MÉTODOS}

Trata-se de um estudo de validação, descritivo, com 4 etapas, sendo 3 do processo de validação e 1 de aplicação dos questionários.

A primeira etapa - validação de conteúdo dos questionários por painel de especialistas composto por quatro membros de uma equipe multiprofissional em saúde; a segunda etapa - validação semântica com a participação de quatorze profissional de saúde docentes e quatorze estudantes de saúde; a terceira etapa validação consensual dos questionários pelo painel de especialistas. Por fim, na quarta etapa, os instrumentos validados foram aplicados aos participantes de pesquisa que atenderam aos critérios de inclusão e aceitaram participar do estudo, através da assinatura do Termo de Consentimento Livre e esclarecido (TCLE).

A pesquisa foi realizada entre abril de 2018 e dezembro de 2019 e a coleta dos dados entre outubro e novembro de 2019. Como critérios de inclusão, foram convidados para participar do estudo os profissionais de saúde ativos de um hospital e de uma odontoclínica, e os estudantes com matrícula ativa e maiores de 18 anos dos cursos de graduação de uma instituição de ensino superior especializada em saúde, instituições da Região Metropolitana de Recife.

Foram excluídos do estudo os profissionais de saúde: em transferência do local de trabalho; os profissionais de saúde que participaram do painel de especialistas para a validação de conteúdo; docentes da Instituição de Ensino (IES): em licença médica; em férias; e estudantes: em afastamento por motivos de doença, trancamento de matrícula; licença maternidade; menores de 18 anos, e os que participaram da validação semântica dos questionários.

Os profissionais de saúde foram convidados para participar do estudo através da abordagem presencial pelo pesquisador em seus respectivos locais de trabalho. Os estudantes receberam o questionário e o TCLE, online, através da Plataforma Limesurvey.

Participaram do estudo um total de 110 profissionais de um universo de 205 (sendo 77 profissionais de saúde do hospital e 33 dentistas da odontoclínica) e 175 estudantes de saúde, cerca de $10 \%$ de um universo de 1.760 estudantes da instituição de ensino superior. 
Buscou-se a participação de pelo ao menos $50 \%$ da população total dos profissionais das instituições de saúde e dos estudantes da instituição de ensino. Esse objetivo foi atingido quanto à participação dos profissionais, pois participaram $53,65 \%$. Do universo total de profissionais, $7,31 \%(\mathrm{~N}=15)$ foram excluídos visto que 3 participaram do painel de especialista e outros 12 por motivo de férias e outros afastamentos que atendiam aos critérios de exclusão. O fato de os profissionais terem sido convidados para participar do estudo através da abordagem presencial pelo pesquisador contribuiu para a adesão.

Porém, quanto à participação dos estudantes da IES esse objetivo não foi atingido, participaram 175 (cerca de $10 \%$ do total). Do universo total de estudantes, $0,8(\mathrm{~N}=14)$ foram excluídos devido à participação na etapa de validação semântica dos questionários, o que atendia aos critérios de exclusão.

Essa limitação da pesquisa quanto a adesão dos estudantes deve se ao fato deles terem recebido o questionário e o TCLE, online, através da Plataforma Limesurvey e com prazo de 30 dias para a resposta. Segundo Vieira HC (2010) e colaboradores, entre as desvantagens das pesquisas online, a baixa taxa de resposta aos questionários pode ser considerada a principal. Além disso, o período da coleta coincidiu com o período de estudos para as avaliações finais do semestre letivo (entre novembro e dezembro). Talvez esses fatores, aliados a outros motivos pessoais, tenham contribuído para a não adesão de grande parte dos estudantes.

A pesquisa é resultado da dissertação de mestrado e foi desenvolvida com financiamento próprio, não há conflito de interesse entre os pesquisadores da equipe, e obedeceu aos critérios éticos da Resolução 510/2016 do Conselho Nacional de Saúde/Ministério da Saúde e suas complementares, visando salva guardar os direitos e deveres que dizem respeito à comunidade científica, aos participantes da pesquisa e ao Estado. O projeto da pesquisa foi encaminhado ao Comitê de Ética em Pesquisa da instituição de ensino (CEP/FPS) e somente foi iniciada a coleta de dados da pesquisa após sua aprovação que ocorreu em 28/03/2019, sob o parecer número 3.231.070.

\section{RESULTADOS}

Quanto aos participantes, observa-se que a média de idade entre os 110 profissionais de saúde foi de $37,73$ anos, desvio padrão ( $D P=5,67)$, sendo a maioria $76,36 \%(N=84)$ do sexo feminino e $23,63 \%(N=26)$ do sexo masculino. Essa informação demonstra o número elevado de profissionais de saúde do sexo feminino.

De acordo com dados estatísticos do Instituto Nacional de Estudos e Pesquisas (INEP), sobre o censo da educação superior 2018, a distribuição percentual dos concluintes de graduação, por sexo, demonstra que $75 \%$ dos concluintes dos cursos de saúde e bem estar são do sexo feminino (BRASIL, 2018).

Dentre os profissionais $20,90 \%(N=23)$ são enfermeiros; $8,18 \%(N=9)$ farmacêuticos; $3,63 \%(N=4)$ fisioterapeutas; 2,72\% ( $\mathrm{N}=3$ ) fonoaudiólogos; 26,36\% ( $\mathrm{N}=29)$ médicos; $5,45 \%(\mathrm{~N}=6)$ nutricionistas; $30 \%$ ( $\mathrm{N}=33)$ odontólogos; $1,81 \%(\mathrm{~N}=2)$ psicólogos e $0,90 \%(\mathrm{~N}=1)$ terapeuta ocupacional.

A maioria deles $60,90 \%$ ( $\mathrm{N}=67)$ possuíam o curso de residência/especialização como maior titulação; 26,36\% ( $N=29)$ curso de mestrado; 8,18\% ( $N=9)$ curso de doutorado e 4,54\% ( $N=5)$ curso de graduação.

Quanto aos 175 estudantes de saúde a média de idade foi de 22 anos ( $D P=4,08$ ), sendo a maioria 85,71\% $(\mathrm{N}=150)$ do sexo feminino e $14,29 \%(\mathrm{~N}=25)$ do sexo masculino. Dentre eles $11,43 \%(\mathrm{~N}=20)$ cursavam enfermagem; 9,14\% ( $\mathrm{N}=16)$ cursavam farmácia; 17,14\% ( $\mathrm{N}=30)$ fisioterapia; $42(\mathrm{~N}=74)$ medicina; 7,43\% ( $\mathrm{N}=13)$ nutrição; $12,57 \%$ ( $\mathrm{N}=22)$ psicologia, e não teve a adesão de estudantes de odontologia, provavelmente pelo fato desse curso ter sido iniciado recentemente na instituição, e os estudantes, por estarem no primeiro período, podem não ter apresentado interesse.

No tocante acúmulo de medicamentos em residências, é considerado um dos fatores que contribui para o descarte inadequado desses produtos. Principalmente, devido à falta de obrigatoriedade de recolhimento desses resíduos pelos estabelecimentos farmacêuticos, mais especificamente pelas farmácias e drogarias, contribuindo em muito com consequências desastrosas ao meio ambiente (FERREIRA CL, et al., 2015).

A fim de verificar o comportamento relacionado ao acúmulo de medicamentos em residência e avaliar o conhecimento sobre a forma correta de descarte desse tipo de resíduo por parte dos profissionais e 
estudantes, foi perguntado aos estudantes de saúde: Você possui medicamentos vencidos ou sobras de tratamentos em sua residência que não estejam sendo utilizados? Também foi perguntado: Você faz um controle do prazo de validade e do aspecto físico dos medicamentos armazenados em sua residência? (Tabela 1).

Além disso, foi perguntado aos profissionais e estudantes, como eles descartam os medicamentos vencidos ou sobras de tratamento em seus domicílios?

Seja por desconhecimento ou por falta de informações, semelhante ao que ocorre com grande parte da nossa população, que descarta medicamentos de forma inadequada, a maioria dos profissionais e estudantes de saúde, também disseram que descartam medicamentos de forma incorreta, em pias ou tanques, vasos sanitários, esgoto, e principalmente no lixo comum (BATISTA RS, et al., 2009; HOPPE TR e ARAÚJO LEB, 2012; DINARDI AJ e ARAÚJO SC, 2017).

Tabela 1 - Acúmulo de medicamentos em residência, controle do prazo de validadee destino das sobras dadas pelos entrevistados.

\section{Participantes}

Itens / categorias

\begin{tabular}{cc}
\hline Estudantes & Profissionais \\
$\mathrm{N}=175$ & $\mathrm{~N}=110$
\end{tabular}

\section{Acúmulo de medicamentos em residência por parte}

dos estudantes de saúde

\begin{tabular}{lll}
\hline Sim & $76 \%$ & - \\
Não & $24 \%$ & - \\
\hline
\end{tabular}

Controle do prazo de validade e do aspecto físico dos medicamentos armazenados em residência dos estudantes de saúde

\begin{tabular}{lll}
\hline $\operatorname{Sim}$ & $63,43 \%$ & - \\
Não & $36,57 \%$ & - \\
\hline
\end{tabular}

Destino das sobras de medicamentos dadas pelos

\section{Entrevistados}

Descarta no lixo comum

$62,86 \%$

$53,64 \%$

Descarta no vaso sanitário

$2,86 \%$

$20 \%$

Descarta na pia ou tanque

$0,57 \%$

$4,55 \%$

Devolve na farmácia ou drogaria ou unidade de saúde

$$
12 \%
$$

$15,45 \%$

Dá aos vizinhos, amigos ou parentes para uso

$1,14 \%$

$0 \%$

Outros

$20,57 \%$

$6,36 \%$

Fonte: Bucci FR, et al., 2021.

Quanto ao conhecimento das legislações que norteiam o descarte de medicamentos, observa-se no estudo que grande parte dos profissionais e estudantes não conhecem a Resolução da Diretoria Colegiada no 222, de 28/03/2018, da Agência Nacional de Vigilância Sanitária (ANVISA), que regulamenta as Boas Práticas de Gerenciamento dos Resíduos de Serviços de Saúde, incluindo os medicamentos (BRASIL, 2018). 
Também, a maioria desconhece o Plano de Gestão Integrada de Resíduos Sólidos do Município, previsto no art. 18 e 19 da Lei 12.305 de agosto de 2010, que institui a Política Nacional de Resíduos Sólidos e desconhecem as legislações sanitárias e ambientais vigentes (Tabela 2).

Tabela 2 - Legislações que norteiam o descarte de medicamentos no país.

\begin{tabular}{lcc}
\hline & \multicolumn{2}{c}{ Participantes } \\
\cline { 2 - 3 } Itens / categorias & Estudantes & Profissionais \\
& $\mathrm{N}=175$ & $\mathrm{~N}=110$ \\
\hline
\end{tabular}

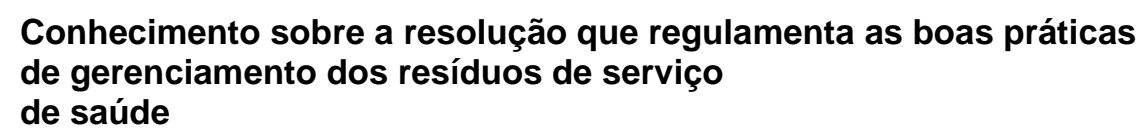

\begin{tabular}{|c|c|c|}
\hline $\operatorname{Sim}$ & $13,71 \%$ & $17,27 \%$ \\
\hline Não & $86,29 \%$ & $82,73 \%$ \\
\hline \multicolumn{3}{|l|}{$\begin{array}{l}\text { Conhecimento sobre o Plano de Gestão Integrada de } \\
\text { Resíduos Sólidos do Município }\end{array}$} \\
\hline Sim & $9,14 \%$ & $5,46 \%$ \\
\hline Não & $90,86 \%$ & $94,54 \%$ \\
\hline $\begin{array}{l}\text { Conhecimento sobre as legislações sanitárias e } \\
\text { ambientais vigentes }\end{array}$ & & \\
\hline Sim & $12,57 \%$ & $12,73 \%$ \\
\hline Não & $87,43 \%$ & $87,27 \%$ \\
\hline
\end{tabular}

Fonte: Bucci FR, et al., 2021.

Outro aspecto importante abordado na pesquisa, refere-se ao recebimento de instruções ou treinamentos sobre descarte correto de medicamentos durante os cursos de graduação. Para evidenciar esse aspecto foi perguntado: Durante a graduação você recebeu instruções ou treinamentos conforme as legislações sanitárias e ambientais vigentes sobre descarte correto de medicamentos vencidos ou inutilizados?

A maioria dos profissionais de saúde $53,63 \%(\mathrm{~N}=59)$ dizem que não recebeu instruções ou treinamentos durante a graduação; outros $15,45 \%(N=17)$ responderam que não lembram; e somente $30,90 \%(N=34)$ disseram que receberam instrução. De modo semelhante, a maioria dos estudantes $70,86 \%(\mathrm{~N}=124)$ dizem ainda não ter recebido instruções ou treinamentos durante a graduação e a minoria deles $29,14 \%(N=51)$ dizem já ter recebido.

Além disso, perguntou se aos profissionais de saúde: Durante a vivência profissional, você recebeu instruções ou treinamentos conforme as legislações sanitárias e ambientais vigentes sobre descarte correto de medicamentos vencidos ou inutilizados?

Observa-se que a maioria dos profissionais 55,45\% ( $\mathrm{N}=61)$ afirmam não ter recebido; 7,27\% ( $\mathrm{N}=8)$ dizem não lembrar; e somente $37,27 \%(\mathrm{~N}=41)$ afirmam que receberam instruções ou treinamentos durante a vivência profissional.

\section{DISCUSSÃO}

Comportamento semelhante foi identificado em outros estudos, nos quais buscou se identificar se os participantes possuíam medicamentos em desuso em suas residências. A maioria dos entrevistados responderam que possuíam medicamentos em suas residências e parte considerável desses não faziam controle da validade (BRUM CA, et al. 2007; HOPPE TR e ARAÚJO LEB 2012; MILANEZ MC, et al. 2013; TAVERA S, et al. 2017).

Ainda, de acordo com Ramos HMP (2017) e colaboradores, em seu estudo que analisou a forma de descarte de medicamentos pela população do Distrito Federal (DF), dentre os 393 entrevistados, $73,8 \%$ 
referiram possuir estoque de medicamentos no seu domicílio, 78,9\% afirmaram já ter realizado o descarte de medicamentos, sendo a maioria no lixo comum $(73,6 \%)$. Assim, evidencia se que o descarte inadequado de medicamentos tem sido uma prática comum pela população.

Possivelmente esse comportamento está relacionado com a facilidade existente em adquirir medicamentos sem prescrições médicas; ao abandono do tratamento por não adesão; desaparecimento momentâneo dos sintomas que levaram ao tratamento; além de sobras de tratamentos anteriores devidos os medicamentos não oferecerem a possibilidade de compra de quantidade fracionada, sendo vendidos em quantidade superior ao necessário (EICKHOFF P, et. al., 2009; HOPPE TR e ARAÚJO LEB, 2012; TAVERA S, et al., 2017).

Cabe salientar quede acordo com Ramos HMO, et al. (2017), a população realiza o descarte de medicamentos de forma inadequada e, em consequência disso, os catadores de lixo acabam sofrendo diversos riscos provocados pela exposição a esses produtos. Ressalta que apesar de o Brasil ter conseguido consideráveis avanços na legislação referente ao descarte de Resíduos de Serviços de Saúde (RSS), ainda persistem lacunas quanto ao descarte desses resíduos gerados em domicílios, bem como na fiscalização da destinação final, e que tais deficiências necessitam ser supridas.

No tocante ao conhecimento das legislações, é de suma importância para os profissionais de saúde e futuros profissionais, uma vez que regulamenta entre outras coisas, que os resíduos gerados pelos serviços de assistência domiciliar devem ser acondicionados, identificados e recolhidos pelos agentes de atendimento ou por pessoa treinada para a atividade e encaminhados ao estabelecimento de saúde de referência (BRASIL, 2018).

Enquanto não for criado um programa em nosso país que seja eficaz para o descarte de resíduos de medicamentos gerados nas residências, é necessário a promoção de ações envolvendo a conscientização da população, através de campanhas informativas sobre o descarte ambientalmente correto, bem como o recolhimento dos medicamentos em desuso, com a colaboração das entidades responsáveis para o descarte adequado (TAVERA S. et al., 2017).

Nesse contexto se inserem os profissionais de saúde, pois devem ser considerados elos fundamentais para a explicação e conscientização da sociedade quanto ao manejo e descarte correto desse tipo de resíduo, considerado nocivo ao meio ambiente e a saúde quando descartado de forma inapropriada.

De acordo com Blankenstein GMP e Junior AP (2018) a educação ambiental e a compreensão das legislações que norteiam o gerenciamento, manejo e descarte de resíduos de medicamentos pode ser o primeiro passo para conhecer a forma prevista para o descarte correto.

No entanto, para que isso seja possível, é preciso que ocorram mudanças no panorama atual, pois no presente estudo evidencia a falta de conhecimento das legislações sanitárias e ambientais pela maioria dos profissionais e estudantes de saúde.

De acordo com Dinardi AJ e Araújo SC (2017), historicamente e ainda na atualidade, em diversos cursos de graduação da área de saúde não há a devida preocupação com a questão ambiental. Ou seja, desde a elaboração dos Projetos Pedagógicos dos Cursos (PPC), a temática socioambiental não é contemplada.

Devido a isso, observa-se fragilidade de informação quanto ao gerenciamento e gestão do descarte dos resíduos de medicamentos, gerando dúvida da população, dos profissionais de saúde e estudantes (futuros profissionais), de onde e como fazer o descarte adequado desses produtos.

Dessa forma, mostra-se necessário que ocorra maior orientação, tanto a população em geral, mas primordialmente aos profissionais e estudantes de saúde, pois são fundamentais para esclarecer e conscientizar as pessoas quanto à destinação final ambientalmente correta desse tipo de resíduo (BATISTA RS, et al., 2009; MAIA HJL, et al., 2013).

Quanto a isso, no presente estudo, a quase totalidade dos estudantes $97,14 \%(\mathrm{~N}=170)$ afirmam ser importante que os cursos de graduação da área de saúde possuam na matriz curricular o tema relacionado à educação ambiental e descarte correto de resíduos de serviços de saúde. 
Não obstante, 92,57\% ( $\mathrm{N}=162)$ também dizem considerar importante que os cursos de graduação da área de saúde possuam na matriz curricular o tema relacionado a descarte de medicamentos vencidos ou sobras de tratamento.

Ademais, de acordo com Lemes EO e Silva JR (2014), a educação ambiental pode ser o caminho para as comunidades montarem seus valores sociais, por meio de conhecimentos, habilidades e atitudes, que ensejam a preservação do meio ambiente. Deve ser considerado um recurso contínuo de aprendizagem, alcançando os diversos ciclos do ensino, tendo a sustentabilidade como fator indispensável para a saúde.

\section{CONCLUSÃO}

O estudo possibilitou a avaliação do conhecimento dos profissionais e estudantes de saúde sobre o descarte adequado de medicamentos por meio de questionários validados tornando possível confirmar na amostra do presente estudo que a maioria dos profissionais e estudantes de saúde não possuem conhecimento sobre descarte adequado de medicamentos. Grande parte deles descartam medicamentos de forma inapropriada principalmente no lixo comum. Ademais, a maioria dos participantes apresentam falta de conhecimento sobrelogística reversa de medicamentos e sobre as legislações que norteiam o descarte de medicamentos no país.

\section{REFERÊNCIAS}

1. ALBANAZ HF, et al. Descarte de Medicamentos: Uma panorâmica da atual situação. Revista Gestão em Foco, 2017.

2. ALVARENGA LSV, NICOLETTI MA. Descarte doméstico de medicamentos e algumas considerações sobre o impacto ambiental decorrente. Revista Saúde. Ung-Ser, 2010; 4(3):34-39.

3. BATISTA RS, et al. Ecologia na Formação do Profissional de Saúde: Promoção do Exercício da Cidadania e Reflexão Crítica Comprometida com a Existência. Revista Brasileira de educação Médica, 2009; 2(33):271-275.

4. BLANKENSTEIN GMP, JUNIOR AP. O descarte de medicamentos e a política nacional de resíduos sólidos: Uma motivação para a revisão das normas sanitárias. Rev. Dir. sanit., 2018;19(1):50-74.

5. BORGES RM, et al. Uso de filtros de carvão ativado granular associado a microrganismos para remoção de fármacos no tratamento de água de abastecimento. Revista Engenharia Sanitária e Ambiental, 2016; 21(4):709-720.

6. BRASIL. Ministério da Saúde. Resolução RDC no 222, de 28 de março de 2018. Regulamenta as boas Práticas de Gerenciamento dos Resíduos de Serviços de Saúde e dá outras providências. Disponível em: www.portal.anvisa.gov.br.documentos.resolucao222. Acesso em: 15 nov. 2018.

7. BRUM CA, et al. Avaliação do estoque de medicamentos das residências da Região do Vale do Aço-MG. Rev. Bras. Farm., 2007; 88(4):173-176.

8. DINARDI AJ, ARAÚJO SC. Inserção da temática ambiental nos cursos de graduação da Universidade Federal do Pampa Uruguaiana - RS. Revista Debates em Educação, 2017; 9(17):59-78.

9. EICKHOFF P, et al. Gerenciamento e destinação final de medicamentos: uma discussão sobre o problema. Revista Bras. Farm., 2009;1(90):64-68.

10. FEITOSA AV, AQUINO MD. Descarte de medicamentos e problemas ambientais: o panorama de uma comunidade no município de Fortaleza/CE. Revista Ciência e Natura, 2016; 38(3):1590-1600.

11. FERREIRA CL, et al. Análise do conhecimento da população sobre descarte de medicamentos em Belo Horizonte Minas Gerais. Revista Interfaces Científicas - Saúde e Ambiente, 2015; 3(2):9-18.

12. GUERRIERI FM, HENKES JA. Análise do descarte de medicamentos vencidos: Um estudo de caso no município de Rio das Ostras (RJ). Revista gest. sust. Ambient., 2017; 6(1):566-608.

13. HOPPE TR, ARAÚJO LEB. Contaminação do meio ambiente pelo descarte inadequado de medicamentos vencidos ou não utilizados. Revista Monografias ambientais, 2012; 6(6):1249-1262.

14. LEMES EO, SILVA JR. Gestão ambiental: Educação ambiental sobre o descarte de embalagens de medicamentos no município de Anápolis - Goiás. Magistro de filosofia ano VII, 2014; 14(1):87-96.

15. MAIA HJL, et al. Educação ambiental: instrumento de mudança de percepção ambiental de catadores de materiais recicláveis organizados em associação. Revista Monografias Ambientais (REMOA), 2013; 13(13):2797-2806.

16. MILANEZ MC, et al. Avaliação dos estoques domiciliares de medicamentos em uma cidade do Centro-Sul do Paraná. Revista de Ciências Médicas e Biológicas, 2013; 12(3):283-289.

17. OLIVEIRA NR. A relevância da prescrição e do uso racional de medicamentos para mitigar potenciais riscos à saúde e ao meio ambiente. $2015 ; 110$ p.

18. RAMOS HMP, et al. Descarte de medicamentos: uma reflexão sobre os possíveis riscos sanitários e ambientais. Revista Ambiente \& Sociedade, 2017; 10(4):149-174.

19. TAVERA S, et al. Conhecimento de estudantes universitários sobre descarte de medicamentos. Revista Intellectus, 2017; 1(42):84-101.

20. VIEIRA HC, et al. O uso de questionários via e-mail em pesquisas acadêmicas sob a ótica dos respondentes. XIII SEMEAD., 2010. 